\title{
Identification of the Factors Responsible for the Interaction of Hsp90a and its Client Proteins
}

\author{
Ashutosh Shukla and Subhankar Paul
}

\author{
Structural Biology and Nanomedicine Laboratory, Department of Biotechnology and Medical Engineering, National \\ Institute of Technology Rourkela, Rourkela, Odisha, India
}

\begin{abstract}
Hsp90 $\alpha$ is a stress protein that acts as a molecular chaperone and is known to assist in the maturation, folding and stabilization of various cellular proteins known as 'client proteins'. However, the factors that drive the interaction between Hsp90 $\alpha$ and its client proteins are not well understood. In the present investigation, we predicted the basis of the different interaction of Hsp90 $\alpha$ with both wild and mutant p53 and other client proteins. We have predicted that the presence of hydrophobic patches having substantial value of hydropathy index and a minimum percent similarity of hydrophobic patches between Hsp90 $\alpha$ and its client proteins of $40 \%$ is a necessary condition for client proteins to be recognized by Hsp90 $\alpha$. We also predicted that the overall percentage hydrophobicity of client proteins more than 20 is a required condition for them to bind with Hsp90 $\alpha$. The docking energy of p53 with Hsp90 $\alpha$ and with multi-chaperone complex was also separately reported. We have reported from docking result that mutant p 53 has a stronger interaction with Hsp90 when associated with multi-chaperone complex than wild type p53 and this might be one of the causes of breast cancer pathogenesis.
\end{abstract}

Keywords: p53, hydrophobic patches, hydropathy index, docking, surface hydrophobicity plot.

\section{INTRODUCTION}

The molecular chaperone Hsp90 (heat-shock protein 90) is a highly conserved, essential and abundantly available (available 1-2\% of total cellular protein), homo dimeric molecular chaperone found in the eukaryotic cells [1, 2]. Hsp90 is found in two isoforms; Hsp90 $\alpha$ and Hsp90 $\beta$ [3]. The Hsp $90 \alpha$ has been found to be stress inducible and overexpressed in many cancer cells [3]. It is an essential heat shock protein that is involved in the maturation and activation of number of proteins known as client proteins [4]. Hsp90 $\alpha$ was also found to be involved in the maturation and stabilization of wide range of oncogenic client proteins that are crucial for oncogenesis and malignant progression $[5,6]$. A large number of Hsp90 $\alpha$ client proteins like transmembrane tyrosine kinases such as Her-2, metastable signaling proteins like Akt, , transcription factor like p53, chimeric signaling proteins like Bcr-Abl, cell cycle regulators ( $\mathrm{Cdk} 4, \mathrm{Cdk} 6)$, and steroid receptors (androgen, estrogen, and progesterone receptors) have been shown to be wrongly regulated in cancer [7].

In cancer cells, Hsp90 $\alpha$ is mostly present as a multichaperone complex whereas non-cancerous cells, they present in an uncomplex state [8]. Hsp90 $\alpha$, despite its ubiquitous nature and importance for cellular viability, has become an important target for cancer therapeutics. Functionally, it does not work alone but it is dependent upon a group of cochaperones in the client protein maturation in an

*Address correspondence to this author at the Department of Biotechnology and Medical Engineering, National Institute of Technology Rourkela, Rourkela, Odisha, India; Tel: +(91)-661-246-2284;

E-mail: spaul@nitrkl.ac.in,
ATP-dependent manner [9]. However, the mechanism by which Hsp90 $\alpha$ selectively binds to various client proteins has not been well understood [10]. Thus, disrupting the complex formed by Hsp90 $\alpha$ and its specific client proteins in cancer cells has been considered to be a potential therapeutic approach.

It is a multi-domain protein consisting of three domains: an N-terminal ATP-binding domain $(\mathrm{N})$; a middle domain (M); and a C-terminal homo dimerization domain (C) containing a tetratricopeptide repeat (TPR) protein binding motif. Its interaction with client proteins is regulated by a number of co-chaperones. Association of co-chaperones and client proteins normally regulates the ATPase activity of Hsp90 $\alpha$ [11-14] which triggers the chaperone activity of

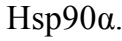

Although the whole mechanism of Hsp90a-mediated folding and maturation of client proteins is not yet clear, some information has already been reported by Felts and his group [15]. A complex process which involves Hsp90 $\alpha$, Hsp70, Hsp40 and HoP (in Human) has been documented by many groups [15-17].

In the present communication, the interaction between Hsp90 $\alpha$ and co chaperones like p23, Aha1, Cdc37, Transcription factor like p53 and kinases like Akt, Ask1, Cdk2, Cdk4, Cdk6, Cdk11, DAPK2, ErbB2, Raf-1, B-Raf was investigated. We showed that client proteins and cochaperones contain hydrophobic patches and hence, they are prone to bind with Hsp90 $\alpha$ through hydrophobic interaction to the hydrophobic patches present in different domains $(\mathrm{C}$ Terminal, N Terminal, Middle domain) of Hsp90 $\alpha$. We also concluded from our results that the percent similarity of sequence of hydrophobic patches between Hsp90 $\alpha$ and its cli- 
ent proteins should have a cutoff value of $40 \%$ and this was the necessary condition for client proteins to be recognized by Hsp90 $\alpha$. Through surface hydrophobicity plot, hydrophobic patches of Hsp90 $\alpha$ and its clients were identified. It was also found that a cut off of $20 \%$ overall hydrophobicity of client proteins was required for being recognized by Hsp90a.Using the Hex docking software it was demonstrated that mutant p53 has a higher affinity to Hsp90 $\alpha$ when it exists in multi-chaperone complex.

\section{METHODS}

Bioinformatics Tools Used for Prediction of Hydrophobic Patches in $H s p 90 \alpha$ Client Proteins and Interacting Amino Acid Sequences Between Hsp90 and its Client Proteins in Human

Amino acid sequence of proteins: Amino acid sequences of Hsp90 $\alpha$, its client proteins and p53 mutant proteins were retrieved from SWISS PROT. The two p53 mutants are found in breast cancer cell lines MCF-7 and T47D.

Retrieval of 3-D protein structure from protein data bank (PDB): We have retrieved 3-D structure of proteins from protein data bank (PDB) which has a fixed PDBid. Human wild type p53 having PDBid-2OCJ, mutant p53 (N239Y) having PDBid-2QXC and mutant p53 (N235Y) having PDBid-2QXB and Human Hop-Hsp70 complex having PDBid-1ELW are retrieved from protein data bank (PDB).

Identification of Hydrophobic patches by Surface Hydrophobicity plot: Kyte-Doolittle is an online software widely applied scale for determining hydrophobic character of a protein [18]. Regions with values above ' 0 ' are hydrophobic in character. Setting window size to 5-7 is suggested to be a good value for finding putative surface-exposed regions. Window size refers to the number of amino acids examined at a time to determine a point with hydrophobic character.

Estimation of Hydropathy index using grand average of hydropathy (GRAVY) Value by ProtParam Tool: ProtParam is a tool which allows the computation of various physical and chemical parameters for a given protein stored in Swiss-Prot or TrEMBL or for a user entered protein sequence [18]. The computed parameters include molecular weight, theoretical pI, atomic composition, extinction coefficient, estimated half-life, instability index, aliphatic index and grand average of hydropathicity (GRAVY). The GRAVY value of a protein here was calculated by adding of hydropathy values of all the amino acids divided by the number of residues in the sequence.

Estimation of Overall percentage of Hydrophobicity using SAPS (Statistical Analysis of Protein Sequences): SAPS evaluates a wide variety of protein sequence properties by statistical criteria [19]. Properties that were considered include compositional biases, clusters and runs of charge and other amino acid types, different kinds and extents of repetitive structures, locally periodic motifs, and anomalous spacings between identical residue types. Overall percentage of hydrophobicity was defined as the ratio of $x / n$, where ' $x$ ' is the number of amino acids having high hydro- phobicity value ( $\mathbf{L}, \mathbf{V}, \mathbf{I}, \mathbf{F}, \mathbf{M})$ and 'n' is the total number of amino acids in the sequence.

Similarity of hydrophobic patches between Hsp90a and its client protein: Percent similarity of hydrophobic patches between Hsp90 $\alpha$ and its client protein was calculated using Ident and Sim tool. In this study, amino acid interaction of a similar kind (Hydrophobic- Hydrophobic, identical charged-charged) was allowed.

Prediction of 3-dimensional protein structures of by ITASSER (Iterative Threading ASSEmbly Refinement program): It predicts the 3 dimensional structure of protein based on Confidence score and TM-Score. We have modeled middle domain of Hsp90 $\alpha$ through I-TASSER software (Online 3-D Protein Structure Prediction) having C Score 1.59 and TM-Score 0.94+-2.4.

Protein- Protein Interactions by Hex5.0: Hex is an interactive molecular graphics program developed by Dave Ritchie for estimating docking energy [20]. It is very useful for docking of protein and ligand molecules. Hex 5.0 carries out all forms of docking such as rigid, flexible and semi flexible docking. It calculates the intermolecular "energies" by adding up all intermolecular interactions (e.g. Vander Waals, electrostatic) that occur between a ligand and protein targe. Hex Server has an easy-to-use form-based interface, through which users may upload a pair of protein structures in PDB format through Hex software. We have performed docking between middle domain of human Hsp90 and wild type p53 as well as both mutant p53. The "Final Search" entry box is used to specify the main expansion order $\mathrm{N}$. Although the default value of $\mathrm{N}=25$ is usually applied. All the proteins were docked for 100 conformations. The docking energy was calculated. The lowest energy was reported to be the fit as it implies the strongest affinity and stability.

\section{RESULTS}

Identification of hydrophobic patches in human Hsp90a and its co-chaperone: Hydrophobic patches present in Hsp90 $\alpha$ and its cochaperone (p23, Aha1, Cdc37 and Hsp70) were identified through Surface Hydrophobicity Plot with Kyte-Doolittle scale and using a window size 7. We found three different hydrophobic patches present in Hsp90, $\mathrm{N}$-Terminal, C-Terminal and Middle domain. Hydrophobic patches and its location in Hsp90 $\alpha$ cochaperones were also predicted (Table 1).

Identification of hydrophobic patches in Hsp90a Client Proteins (Transcription Factor): Hydrophobic patches present in Hsp90 $\alpha$ client protein p53 (Transcription Factor) were also identified. These patches were present in Middle domain of p53 (Table 1) and we also calculated \% overall hydrophobicity and hydropathy index of Hsp90 (Table 2).

Hydropathy index of Hsp90 $\alpha$ was also estimated according to the grand average of hydropathy (GRAVY) Value by ProtParam Tool and shown in (Table 2).

Interaction of p23, Aha1 and Cdc37 with Hsp90a: A cut-off value of the interaction between $\mathrm{Hsp} 90 \alpha$ (predicted hydrophobic patches) and the binding site of its cochaperones on the Hsp90 $\alpha$ protein [\% Similarity] was considered to be $40 \%$. Based on our assumption we have 
Table 1. Predicted Hydrophobic Patch in Hsp90a and its co-chaperone in human

\begin{tabular}{|c|c|c|}
\hline \multirow{2}{*}{ Hsp90a } & AFQAEIAQLMSLIINTFYSNKEIF & N Terminal \\
\hline & KNDKSVKDLVILLYETALLSSGF & C Terminal \\
\hline \multicolumn{3}{|c|}{ Co-chaperones } \\
\hline p23 & NFEKSKLTFSCLGGSDNFKHLNE & N Terminal \\
\hline Cdc37(p50) & EQVAHQTIVMQFILELAKSLKVDP & C Terminal \\
\hline Harc & QNHVPHSGVGSIGLLESLPQNPDYL & C Terminal \\
\hline
\end{tabular}

Table 2. Hydropathy Index of Predicted Hydrophobic Patch in Hsp90a in human

\begin{tabular}{|c|c|c|}
\hline Chaperone & \% Overall Hydrophobicity & Hydropathy Index of the predicted patch \\
\hline \hline Hsp90a & $26.6 \%$ & 2.029 (LMSLIIN), 2.100 (FRALLFV), 2.186 (DLVILLY) \\
\hline
\end{tabular}

identified a hydrophobic patch "TFSCLG" located in the N terminal domain of p23 which interacted to N-terminal domain of Hsp90 $\alpha$ (Table 3) and the value of \% similarity was 42.86. Similarly, it was observed that in the N terminal domain (1-153) of Aha1, a hydrophobic patch "VEISVSL" was identified with a \% similarity value of 42.86 which interacted with the middle domain of Hsp90 $\alpha$ (Table 3). A hydrophobic patch "VMQFIL" having a percent similarity of 57.14 was also identified in the $\mathrm{C}$ terminal domain (138378) of $\mathrm{Cdc} 37$ and this patch was responsible to interact with $\mathrm{N}$ terminal domain of Hsp $90 \alpha$ (Table 3).

Hsp90a binds to wild type p53: We have considered a cut-off value of the interaction between Hsp90 $\alpha$ (predicted hydrophobic patches) and wild type p53 binding site [\% Similarity] was $40 \%$. In the middle domain of wild type $\mathrm{p} 53$,

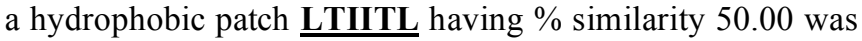
identified which interacted with middle domain of Hsp90 (Table 4).

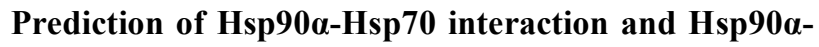
Hsp40 non-interaction: Hydrophobic patch present in middle domain of Hsp70 was predicted which interact with hydrophobic patch of the middle domain of Hsp90 $\alpha$ (Table 4). When hydrophobic patches for the interaction between Hsp90 $\alpha$ and Hsp40 were predicted of three different domains, interestingly the maximum $\%$ similarity was found to be 28.57 and this was much below the cut off value of $40 \%$ (Table 4).

Interaction of various kinases and Hsp90a: The location of the hydrophobic patch in various kinases was also predicted. A cut-off value of the interaction between Hsp90 $\alpha$ (predicted hydrophobic patches) and its kinases binding site on the Hsp90 $\alpha$ protein [\% Similarity] was also considered as $40 \%$. Based on our assumption we have identified kinases like Ask1,B-Raf,Raf-1 having a hydrophobic patch "VQVVLFG" (C terminal domain), "FGIVLY" (C terminal domain), "VGIVLYE" (C terminal domain), respectively (Table 4) which interact with middle domain of Hsp90 (Table 4) and the maximum value of \% similarity was 71.43 . Similarly, when the interaction of other kinases like Akt, Cdk2, Cdk4, Cdk6, Cdk11, DAPK2, ErbB2 with the middle domain of Hsp90 $\alpha$ was monitored, the value of \% similarity was observed to be $50.00 \%$ (Table 5). We have identified hydrophobic patches in kinases and they are shown in Table 5 .

Predicted charged patch of TPR Motif in cochaperones: TPR Motif sequences of Hsp90 $\alpha$ cochaperones were retrieved from SWISS PROT. Charged patches in TPR Motif co-chaperones by surface hydrophobicity plot were predicted (Table 6). Regions with values below 0.0 are hydrophilic in character. Window size was set to 5-7 as it was suggested to be a good value for finding putative surface-exposed regions containing charged patches. Window size refers to the number of amino acids examined at a time to determine a point with hydrophilic character. These charged patches interacted with EEVD on C Terminal domain of Hsp90 $\alpha$. Some TPR Motif of co-chaperones which interacted with EEVD of $\mathrm{C}$ Terminal domain of Hsp90 $\alpha$, did not have charged patch. \% similarity between predicted charged patches in TPR domain of co-chaperones and EEVD on C Terminal domain of Hsp90 $\alpha$ was calculated (Table 7). 
Table 3. Interaction of Co-Chaperones Like p23, Aha1 and Cdc37 with Hsp90a

\begin{tabular}{|c|c|c|c|c|c|c|}
\hline $\begin{array}{l}\text { Hsp90 co- } \\
\text { chaperone }\end{array}$ & $\begin{array}{c}\text { \% Overall } \\
\text { hydrophobic- } \\
\text { ity }\end{array}$ & $\begin{array}{c}\text { Predicted } \\
\text { Hydrophobic } \\
\text { Patch } \\
\text { (Located } \\
\text { Region) }\end{array}$ & $\begin{array}{l}\text { Hydropathy } \\
\text { index of the } \\
\text { predicted } \\
\text { patch }\end{array}$ & $\begin{array}{c}\text { \% Similarity between } \\
\text { predicted hydrophobic } \\
\text { patch and FRALLFV on } \\
\text { Middle domain of Hsp90 }\end{array}$ & $\begin{array}{c}\text { \% Similarity between } \\
\text { predicted hydrophobic } \\
\text { patch and DLVILLY on } \\
\text { C Terminal domain of } \\
\text { Hsp90 }\end{array}$ & $\begin{array}{c}\text { \% Similarity between } \\
\text { predicted hydrophobic } \\
\text { patch and LMSLIIN on } \\
\mathrm{N} \text { Terminal domain of } \\
\text { Hsp90 }\end{array}$ \\
\hline p23 & $23.1 \%$ & $\begin{array}{c}\text { TFSCLG } \\
\text { (N Terminal) }\end{array}$ & 1.200 & $14.29 \%$ & $28.57 \%$ & $42.86 \%$ \\
\hline $\begin{array}{c}\mathrm{Cdc37} \\
(\mathbf{p 5 0})\end{array}$ & $23.8 \%$ & $\begin{array}{c}\text { VMQFIL } \\
\text { (C Terminal) }\end{array}$ & 2.283 & $14.29 \%$ & $28.57 \%$ & $57.14 \%$ \\
\hline $\begin{array}{c}\text { Wild type } \\
\text { p53 }\end{array}$ & $20.6 \%$ & $\begin{array}{l}\text { LTIITL } \\
\text { (Middle } \\
\text { domain) }\end{array}$ & 1.783 & $50 \%$ & $42.86 \%$ & $42.86 \%$ \\
\hline Hsp40 & $24.7 \%$ & $\begin{array}{c}\text { TFASFF, } \\
\text { FDIFFAS } \\
\text { (N Terminal) }\end{array}$ & $1.450,1.867$ & $\begin{array}{l}28.57 \% \\
28.57 \%\end{array}$ & $\begin{array}{l}14.29 \% \\
28.57 \%\end{array}$ & $\begin{array}{l}14.29 \% \\
14.29 \%\end{array}$ \\
\hline
\end{tabular}

Table 4. Predicted Hydrophobic Patch of Hsp90a client proteins (Kinases) in human

\begin{tabular}{|c|c|c|}
\hline Akt & DRARFYGAEIVSALDYLHSEK & Middle domain \\
\hline Cdk2 & SLLKELNHPNIVKLLDVIHTEN & $\mathrm{N}$ Terminal \\
\hline Cdk11 & HKVKPDSKVELLLQKLLTMDP & Middle domain \\
\hline ErbB2 & DGENVKIPVAIKVLRENTSPKANK & Middle domain \\
\hline B-Raf & PYSFQSDVYA $\underline{\text { FGIVLYELMTGQLPYS }}$ & C Terminal \\
\hline DAPK2 & CCYDYFAANDPTSIHVVVFSLEEPYEIQLN & Middle domain \\
\hline
\end{tabular}

Interaction study between Hsp90 $\alpha$ and wild type p53: 3D structures of human wild type p53 (PDBid- 2OCX) as well as mutant p53 (N239Y) having PDB id- 2QXC and mutant p53 (N235Y) having PDB id- 2QXB were retrieved from protein data bank. Middle domain of Human Hsp90 $\alpha$ was docked with wild type p53 as well as with both mutant p53 using Hex software. It was observed that Hsp90 $\alpha$ formed complex with wild type p53 with a docking energy of -636.75 (Etotal) (Table 8 and Fig. 1) while mutant p53 $(\mathrm{N} 239 \mathrm{Y})$ and mutant p53 (N235K) formed complex inmid- dle domain of human Hsp90 $\alpha$ with a docking energy of 484.68 and -399.67 (Etotal), respectively (Fig. 2 \& 3). Table 8 shows docking energy of interaction between various proteins/complexes and p53 molecule.

Strong Interaction between $\mathrm{Hsp90 \alpha}$ and mutant p53 through forming multi-chaperone complex: 3D structure of human Hop-Hsp70 complex having PDB id 1ELW was taken from Protein Data Bank. By Hex5.0 software we have docked the middle domain of human Hsp90 $\alpha$ and 
Table 5. Prediction of Interaction Between Hsp90 and client proteins (Kinases)

\begin{tabular}{|c|c|c|c|c|c|}
\hline $\begin{array}{l}\text { Hsp90 client } \\
\text { proteins } \\
\text { (Kinases) }\end{array}$ & $\begin{array}{c}\% \text { Overall } \\
\text { hydrophobicity }\end{array}$ & $\begin{array}{l}\text { Hydropathy index of } \\
\text { the predicted patch }\end{array}$ & $\begin{array}{c}\text { \% Similarity between } \\
\text { predicted hydrophobic } \\
\text { patch and FRALLFV on } \\
\text { Middle domain of Hsp90 }\end{array}$ & $\begin{array}{c}\text { \% Similarity between } \\
\text { predicted hydrophobic } \\
\text { patch and DLVILLY on } \\
\text { C Terminal domain of } \\
\text { Hsp90 }\end{array}$ & $\begin{array}{c}\text { \% Similarity between } \\
\text { predicted hydrophobic } \\
\text { patch and LMSLIIN on } \\
\mathrm{N} \text { Terminal domain of } \\
\text { Hsp90 }\end{array}$ \\
\hline Akt & $27.5 \%$ & 1.333 & $50.00 \%$ & $42.86 \%$ & $42.86 \%$ \\
\hline Cdk2 & $33.2 \%$ & 1.871 & $57.14 \%$ & $42.86 \%$ & $42.86 \%$ \\
\hline Cdk4 & $30.4 \%$ & 1.914 & $57.14 \%$ & $42.86 \%$ & $28.57 \%$ \\
\hline Cdk6 & $31.0 \%$ & 1.733 & $57.14 \%$ & $42.86 \%$ & $28.57 \%$ \\
\hline Cdk11 & $23.1 \%$ & 1.514 & $57.14 \%$ & $42.86 \%$ & $14.29 \%$ \\
\hline Raf-1 & $27.5 \%$ & $2.5,0.857$ & $\begin{array}{l}57.14 \% \\
71.43 \%\end{array}$ & $\begin{array}{l}42.86 \% \\
57.14 \%\end{array}$ & $\begin{array}{l}42.86 \% \\
42.86 \%\end{array}$ \\
\hline B-Raf & $26.4 \%$ & 2.267 & $71.43 \%$ & $57.14 \%$ & $28.57 \%$ \\
\hline
\end{tabular}

Table 6. Predicted charged patch of TPR Motif in Co-chaperones

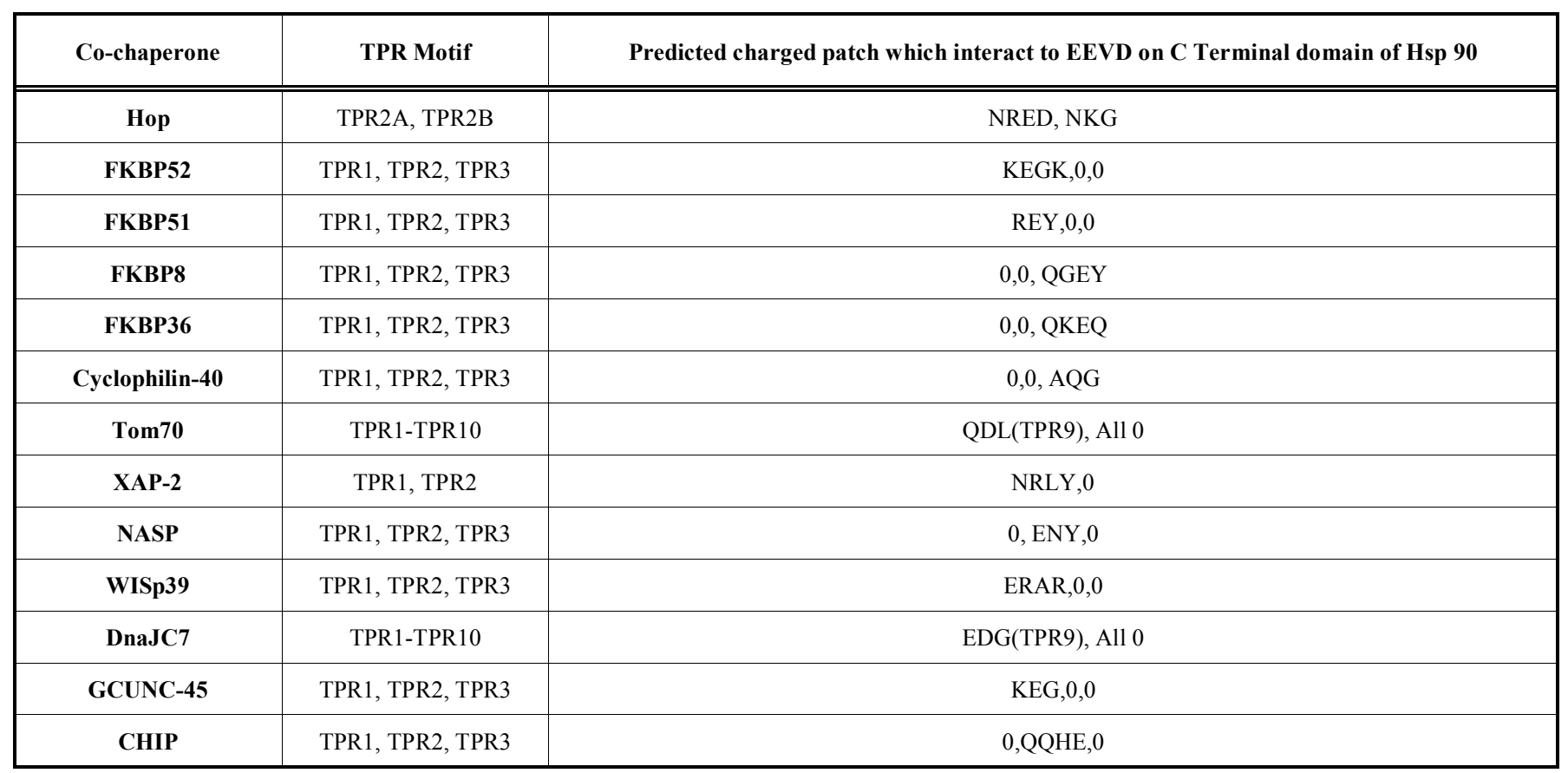

Hop-Hsp70 complex. Further, wild type p53 (PDB id2OCX) was docked with Human Hsp90a-Hop-Hsp70 (middle domain of Hsp90 $\alpha$ ) complex and the docking energy was estimated to be -446.28 (Table 8 and Fig. 4). Similarly, mutant p53 (N239Y) having PDB id- 2QXC and mutant p53 (N235Y) having PDB id- 2QXB were docked with human Hsp90 $\alpha$-Hop-Hsp70 (middle domain of Hsp90) complex which produced a docking energy of -718.16 and -748.12 (Table 8 and Fig. 5 and 6).

\section{DISCUSSION}

In the present investigation, the interaction between Hsp90 $\alpha$ and its various client proteins like p53 and kinases was studied in silico. The presence of single or multiple hydrophobic patches in the amino acid sequence was predicted to be an important factor for client proteins to be recognized by Hsp90 $\alpha$ (Table 1, Table 7). Here, based on our prediction, we concluded that the presence of hydrophobic patches having substantial degree of hydropathy index and percent 
Table 7. Interaction of Hsp90a (Charged Patch EEVD) and its TPR Motif of Co-chaperone (Predicted Charged Patches)

\begin{tabular}{|c|c|c|}
\hline $\begin{array}{l}\text { TPR Motif containing } \\
\text { co-chaperone }\end{array}$ & $\begin{array}{l}\text { TPR Motif Present in } \\
\text { co-chaperone }\end{array}$ & $\begin{array}{l}\text { \% Similarity between predicted Charged patch in TPR domain of co-chaperone and } \\
\text { EEVD on C Terminal domain of Hsp90a }\end{array}$ \\
\hline Hop & TPR2A, TPR2B & $50.00 \%$ (All) \\
\hline FKBP52 & TPR1, TPR2 ,TPR3 & $50.00 \%(\mathrm{TPR} 1)$ \\
\hline FKBP51 & TPR1, TPR2 ,TPR3 & $25.00 \%$ (TPR 1$)$ \\
\hline FKBP36 & TPR1, TPR2 ,TPR3 & $50.00 \%$ (TPR3) \\
\hline Cyclophilin-40 & TPR1, TPR2 ,TPR3 & $50.00 \%$ (TPR3) \\
\hline Tom70 & TPR1- TPR10 & $75.00 \%$ (TPR9) \\
\hline XAP-2 & TPR1, TPR2 ,TPR3 & $50.00 \%(\mathrm{TPR} 1)$ \\
\hline DnaJC7 & TPR1- TPR10 & $75.00 \%$ (TPR7) \\
\hline GCUNC- 45 & TPR1, TPR2 ,TPR3 & $50.00 \%$ (TPR 1$)$ \\
\hline CHIP & TPR1, TPR2 ,TPR3 & $75.00 \%(\mathrm{TPR} 2)$ \\
\hline
\end{tabular}

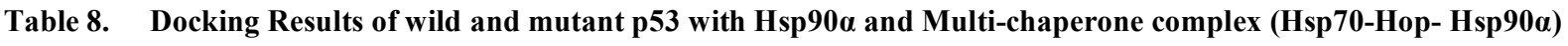

\begin{tabular}{|c|c|c|}
\hline Protein/complex & Hsp90a & Hsp70-Hop-Hsp90a \\
\hline \hline Wild type P53 & -636.75 & -446.28 \\
\hline Mutant P53( N239Y) & -484.68 & -718.16 \\
\hline Mutant p53 ( N235K) & -399.67 & -748.12 \\
\hline
\end{tabular}

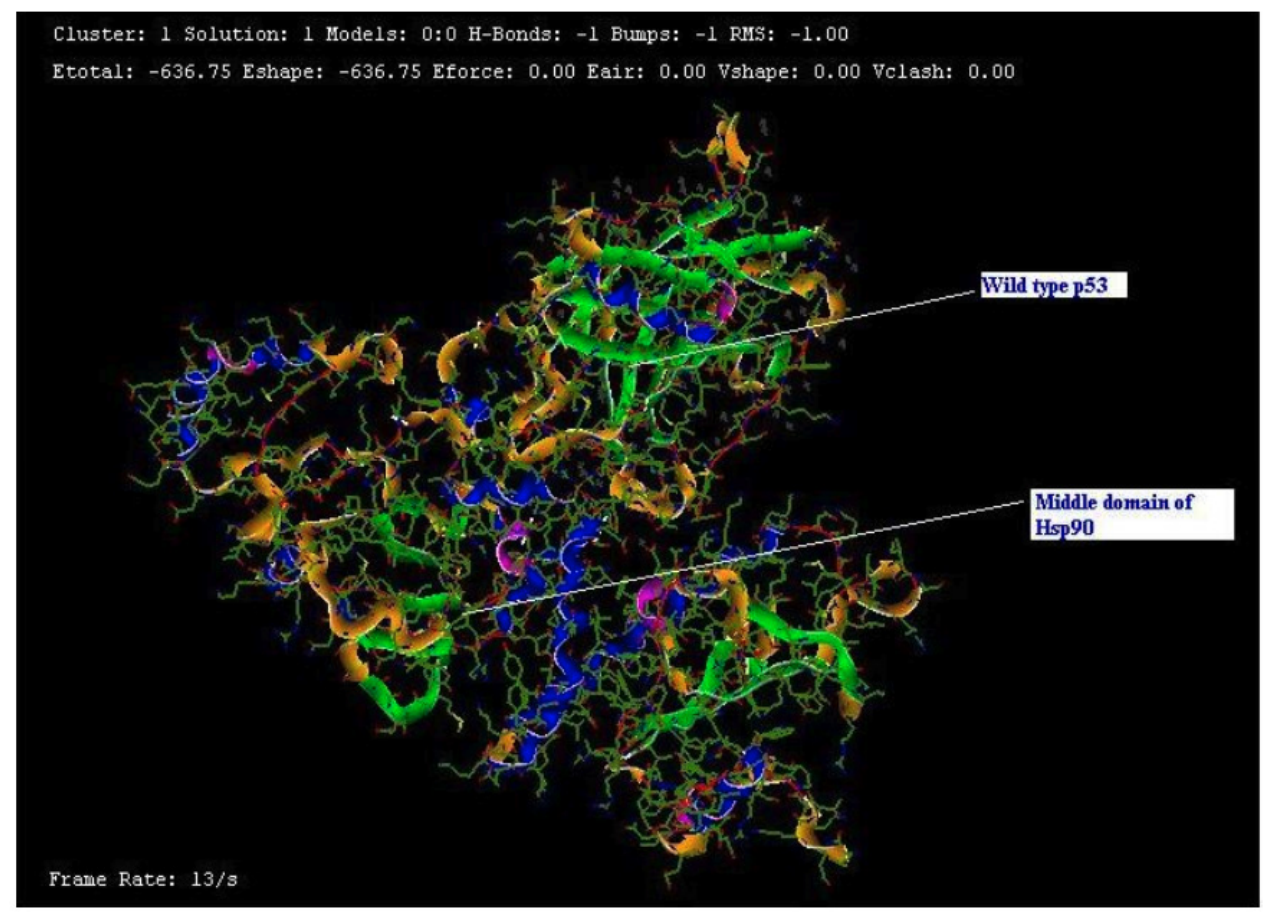

Fig. (1). Hsp90 - wild type p53 complex having docking energy -636.75. 


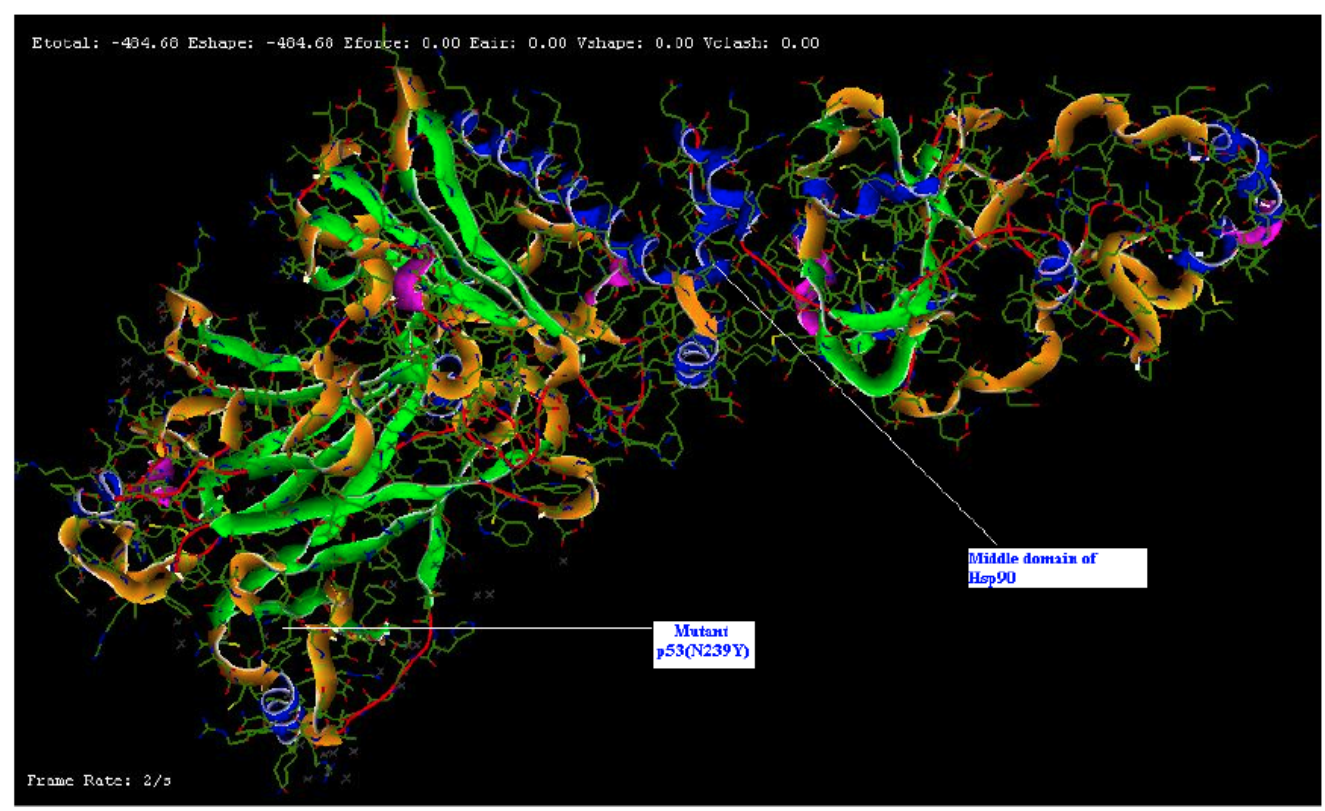

Fig. (2). Hsp90 $\alpha$-mutant p53 (N239Y) complex having docking energy -484.68.

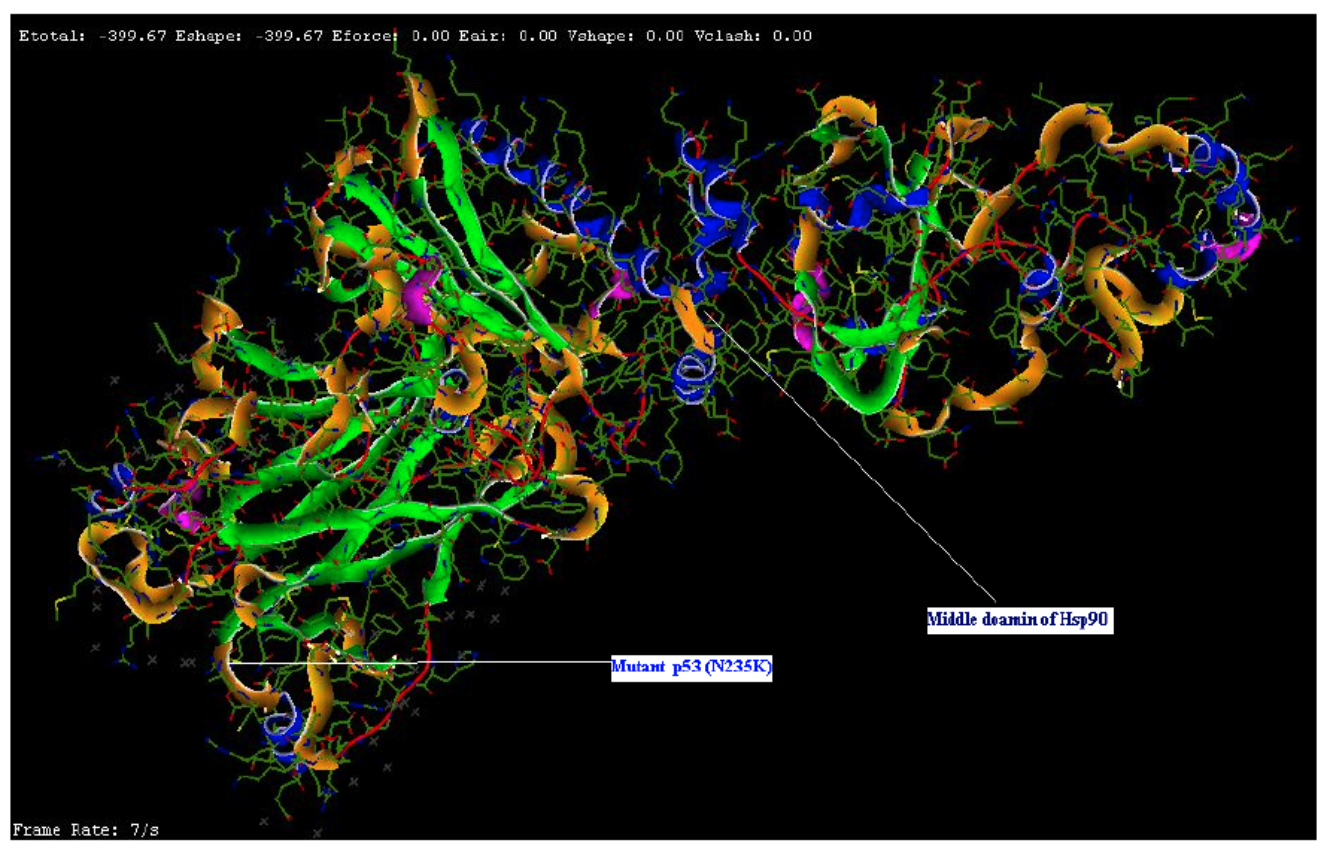

Fig. (3). Hsp90 $\alpha$-mutant p53 (N235K) complex having docking energy -399.67.

similarity of minimum $40 \%$ between hydrophobic patch of Hsp90 $\alpha$ and its client proteins was a necessary condition for client proteins to be recognized by Hsp90 $\alpha$ (Table 3). We also observed that the overall percentage hydrophobicity of these client proteins was more than $20 \%$.

It was also observed that there was $71.43 \%$ similarity in hydrophobic patches between predicted hydrophobic patch in middle domain of Hsp90 $\alpha$ and Hsp70 (Table 3) and this was the highest value among all. The reason was perhaps due to the interaction between two key molecular chaperones Hsp90 $\alpha$ and Hsp70 which work together in cells. Interestingly, the value was only $28.57 \%$ between Hsp90 $\alpha$ and Hsp40. This fact perhaps predicted that Hsp90 $\alpha$ possibly directly interacts with Hsp70 not with Hsp40 or obeys a priority while interacts. Since Hsp40 is a co-chaperone of Hsp70, the result confirmed our agreement.

It is also a well known fact that cellular chaperones and substrate binding take place through hydrophobic interaction $[21,22]$. It was also evident from our results that good amount of hydropathy index was required for a client to interact with Hsp90 $\alpha$. Here we observed that hydropathy index was an important factor to define the binding of client proteins to Hsp90 $\alpha$ because a cut off hydropathy index value of 1.2 was found in all client proteins of Hsp90a (Table 3). However, in case of client proteins like kinases the cut off went up to 1.5 (Table 5) indicating the fact that kinases 


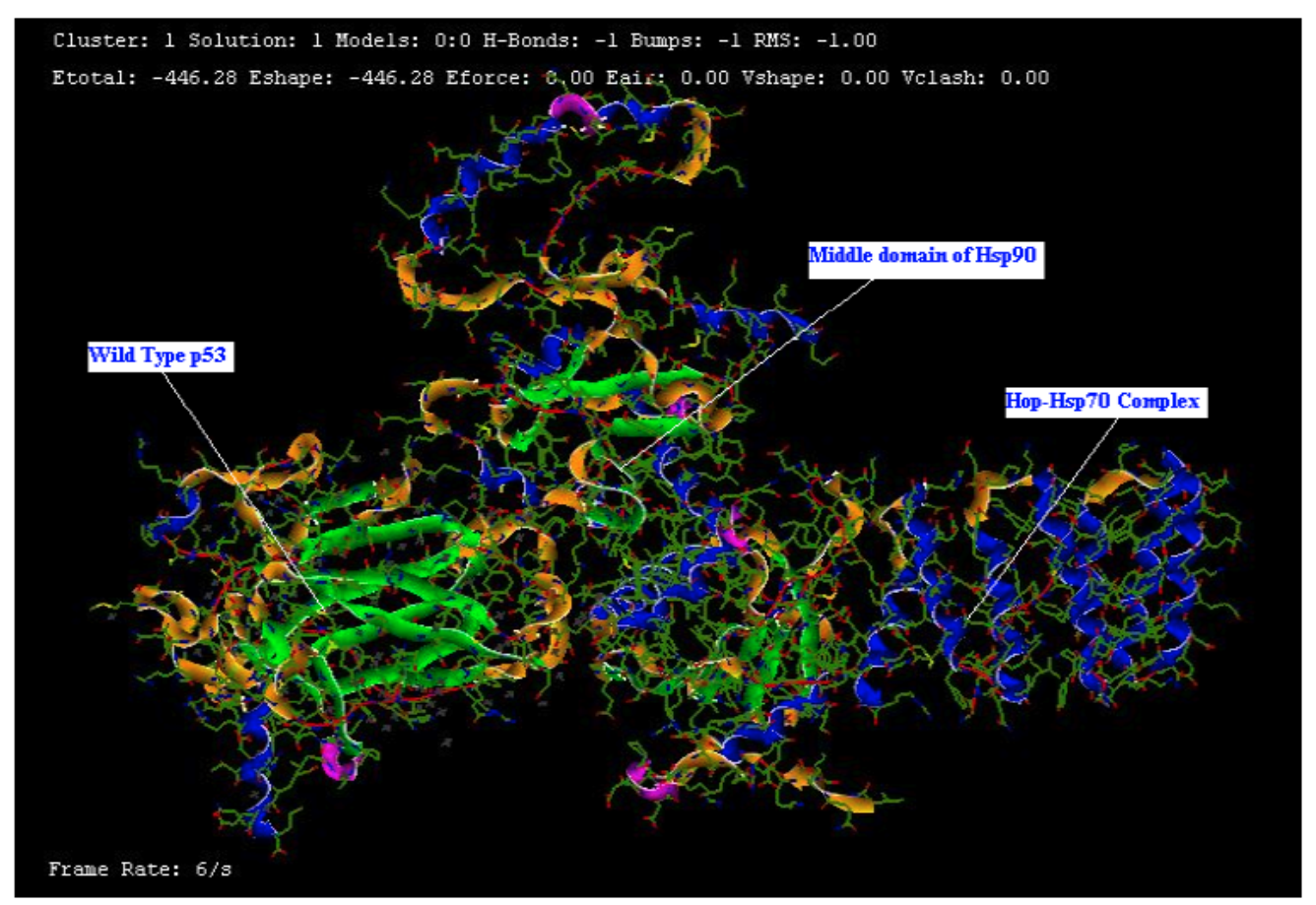

Fig. (4). Hsp70-Hop-Hsp90-wild type p53 complex having docking energy -446.28.

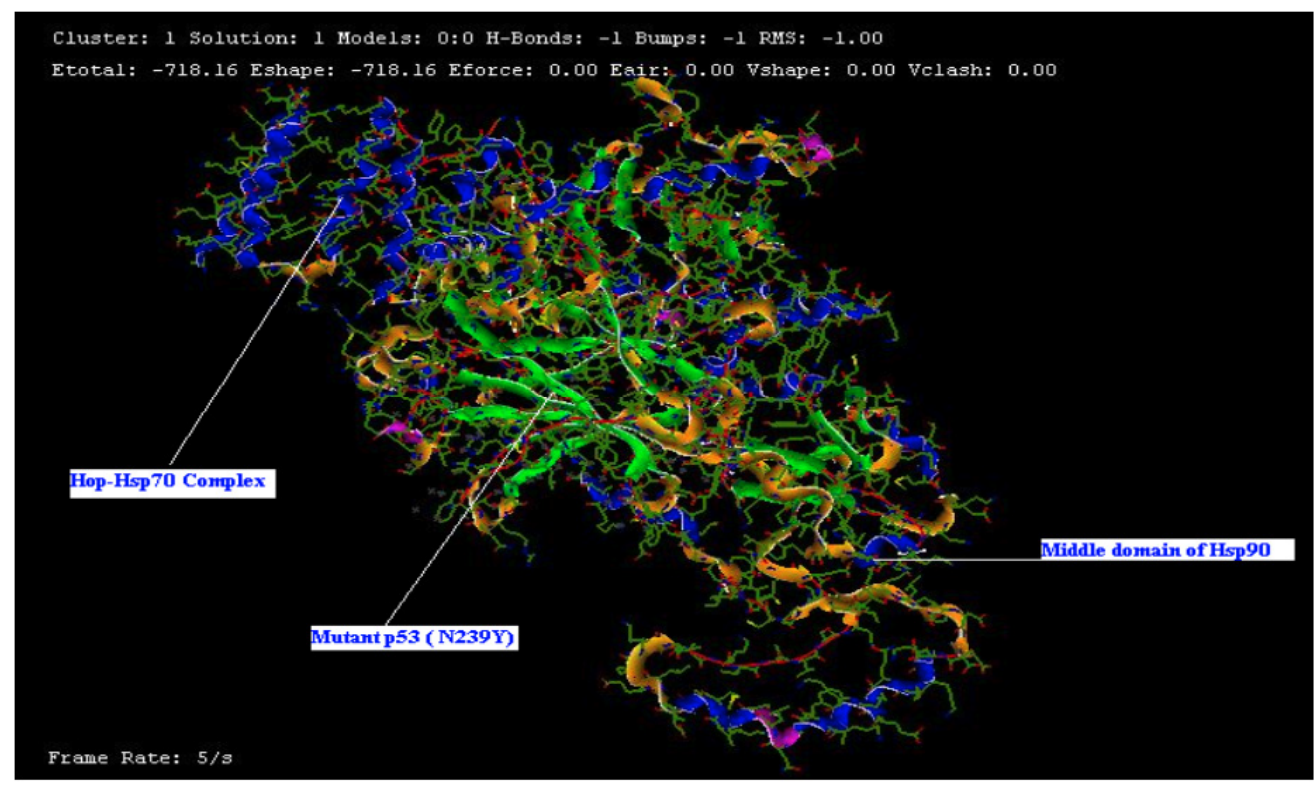

Fig. (5). Hsp70-Hop-Hsp90- mutant p53 (N239Y) complex having docking energy -718.16.

perhaps interact with Hsp90 $\alpha$ strongly and their function and stabilization are highly dependent on Hsp90 $\alpha$ function.

When $\%$ similarity between predicted charged patch in TPR domain of co-chaperone and EEVD on C-Terminal domain of Hsp90 $\alpha$ was estimated, it revealed that in most of the cases the average value was 50\% (Table 7). The high value is indicative of stronger binding affinity and hence cochaperones must be involved in the chaperoning process along with Hsp90 $\alpha$ to work on various client proteins.

The docking energy was also calculated when wild and two different mutant p53 were docked with Hsp90 $\alpha$ by
Hex5.0 software. The similar study was also performed between chaperone complex (Hsp70-HoP-Hsp90 $\alpha$ ) and wild and mutant $\mathrm{p} 53$. The results revealed that wild type p53 has higher binding affinity to Hsp90 $\alpha$ than mutant version p53 (Table 8). More negative value of docking energy indicated higher stability or binding affinity. However, the reverse fact was observed when chaperone complex was considered during docking in place of Hsp90 $\alpha$ alone (Table 8).

Our result helps us to understand that the chaperoning function of Hsp90 $\alpha$ completely depends on the formation of multi-chaperone complex which perhaps triggers a conformational change in Hsp90 2 . This change possibly creates 


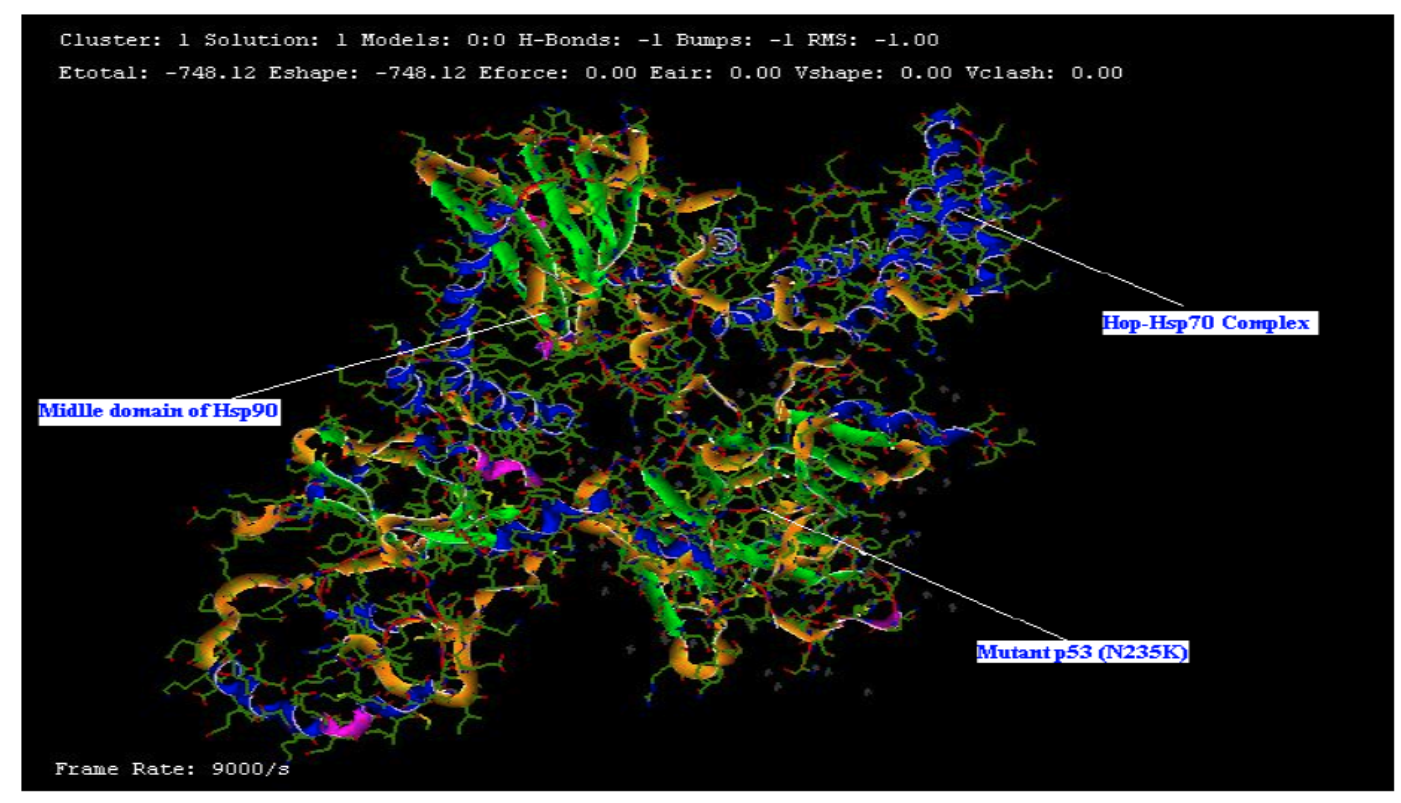

Fig. (6). Hsp70-Hop-Hsp90- Mutant p53 (N235K) complex having docking energy -748.12.

significantly high amount of hydrophobic patches on its surface which further drives various client proteins to bind with Hsp90 $\alpha$. And in the similar way p53 binds with Hsp90a. However, mutant $\mathrm{p} 53$, due to the mutational effect also creates high amount of surface hydrophobicity (that normally exists in misfolded state) and that is the reason why the binding energy of mutant p53 and Hsp90 $\alpha$ interaction in chaperone complex was found higher than wild type p53.

\section{CONCLUSION}

It was clear from our investigation that a minimum/threshold level of overall hydrophobicity $(20 \%)$ and hydropathy index (minimum 1.2) was required for client proteins to be recognized by Hsp90 $\alpha$. The minimum percent similarity of hydrophobic patches between human Hsp $90 \alpha$ and its client protein was found to be $40 \%$ and this was concluded to also be a necessary condition for client protein to be recognized by Hsp90 $\alpha$. From our molecular docking results we concluded that when Hsp90 $\alpha$ was in free state (not in chaperone complex), wild p53 showed higher binding affinity than mutant p53 and the reverse was observed when Hsp90 $\alpha$ was in chaperone complex. The fact indicates that complex formation between Hsp90 $\alpha$ and client protein as well as co-chaperones is essential for a successful chaperone action by Hsp90 $\alpha$ on the client proteins.

\section{CONFLICT OF INTEREST}

The author(s) confirm that this article content has no conflicts of interest.

\section{ACKNOWLEDGEMENT}

The authors thankfully acknowledge the financial support from National Institute of Technology Rourkela and Department of Science and Technology, Government of India for carrying out this work.

\section{REFERENCES}

[1] D. Picard, "Heat-shock protein 90, a chaperone for folding and regulation", Cell. Mol. Life. Sci., vol. 59, pp. 1640-8, 2002.

[2] C. Soti, Z. Nagy, L. Giricz, P. Vigh, P. Csermely, and P. Ferdinandy, "Heat shock proteins as emerging therapeutic targets", $\mathrm{Br}$. J. Pharmacol., vol. 146, pp. 769-780, 2005.

[3] S. H. Millson, A. W. Truman, A. Rácz, B. Hu, B. Panaretou, J. Nuttall, M. Mollapour, C. Söti, and P. W. Piper, "Expressed as the sole Hsp90 of yeast, the alpha and beta isoforms of human Hsp90 differ with regard to their capacities for activation of certain client proteins, whereas only Hsp90beta generates sensitivity to the Hsp90 inhibitor radicicol", FEBS. J., vol. 274(17), pp. 4453-63, 2007.

[4] L. Whitesell, S. L. Lindquist, "HSP90 and the Chaperoning of Cancer", Nat. Rev. Cancer, vol. 5, pp. 761-772, 2007.

[5] A. Kamal, M. F. Boehm, F. J. Burrows, "Therapeutic and diagnostic implications of Hsp90 activation", Trends Mol. Med., vol. 10, pp. 283-290, 2004.

[6] M. V. Powers, and P. Workman, "Targeting of multiple signalling pathways by heat shock protein 90 molecular chaperone inhibitors", Endocr. Relat. Cancer, vol.13, S125-35, 2006.

[7] Y. Li, T. Zhang, "New developments in Hsp90 inhibitors as anticancer therapeutics: Mechanisms, clinical perspective and more potential”, Drug Resis. Update, vol. 12, pp. 17-27, 2009.

[8] G. Chiosis, and L. Neckers, "Tumor selectivity of Hsp90 inhibitors: the explanation remains elusive", ACS Chem. Biol., vol. 1, pp. 279284, 2006.

[9] W. M. J. Obermann, H. Sondermann, A. A. Russo, N. P. Pavletich, and F. U. Hartl, "In vivo function of Hsp90 is dependent on ATP binding and ATP hydrolysis", J. Cell. Biol., vol. 143, pp.901-910, 1998.

[10] M. P. Mayer, C. Prodromou, and J. Frydman. "The Hsp90 mosaic: a picture emerges", Nat. Struct. Mol. Biol., vol. 16, pp. 2-6, 2009.

[11] S. H. McLaughlin, H. W. Smith, and S.E. Jackson, "Stimulation of the weak ATPase activity of human Hsp90 by a client protein. $J$. Mol. Biol., vol. 315, pp. 787-798, 2002.

[12] K. Richter, P. Muschler, O. Hainzl, J. Reinstein, J. Buchner, "Sti1 is a non-competitive inhibitor of the Hsp90 ATPase. Binding prevents the N-terminal dimerization reaction during the ATPase cycle", J. Biol. Chem., vol. 278, pp. 10328-10333, 2003.

[13] G. Siligardi, B. Panaretou, P. Meyer, S. Singh, D. N. Woolfso, P. W. Piper, L. H. Pear, and C. Prodromou, "Regulation of Hsp90 ATPase activity by the co-chaperone Cdc37p/p50cde37”, J. Biol. Chem., vol. 277, pp. 20151-20159, 2002. 
[14] W. Zhang, M. Hirshberg, S. H. McLaughlin, G. A. Laser, J. G. Grossmann, P. R. Nielsen, F. Sobott, C. V. Robinson, S. E. Jackson, and E. D. Laue, "Biochemical and structural studies of the Interaction of Cdc37 with Hsp90", J. Mol. Biol., vol. 340, pp. 891907, 2004.

[15] S. J. Felts, L. M. Karnitz, and D. O. Toft, "Functioning of the Hsp90 machine in chaperoning checkpoint kinase I (Chk1) and the progesterone receptor (PR)", Cell. Stress. Chaper., vol. 12, pp. 353-363, 2007.

[16] M. P. Hernandez, A. Chadli, and D. O. Toft, "HSP40 binding is the first step in the HSP90 chaperoning pathway for the progesterone receptor", J. Biol. Chem., vol. 277, pp. 11873-11881, 2002.

[17] H. Kosano, B. Stensgard, and M. C. Charlesworth, "The assembly of progesterone receptor-hsp90 complexes using purified proteins", J. Biol. Chem., vol. 273, pp. 32973-32979, 1998.

[18] J. Kyte, and R. F. Doolittle, "A simple method for displaying the hydropathic character of a protein", J. Mol. Biol., vol. 157, pp. 105$32,1982$.
[19] V. Brendel, P. Bucher, I. R. Nourbakhsh, B. E. Blaisdell, and S. Karlin, "Methods and algorithms for statistical analysis of protein sequences", Proc. Natl. Acad. Sci. USA, vol. 89, pp. 2002-2006, 1992.

[20] G. Macindoe, L. Mavridis, V. Venkatraman, M. D. Devignes, and D. W. Ritchi, "HexServer: an FFT-based protein docking server powered by graphics processors", Nucleic. Acid Res., vol. 38 , W445-9. 2010.

[21] F. U. Hartl, and M. Hayer-Hartl, "Converging concepts of protein folding in vitro and in vivo", Nat. Struct. Mol. Biol., vol. 16, pp. 574-581, 2009

[22] G. W. Farr, K. Furtak, M. B. Rowland, N. A. Ranson, H. R. Saibil, T. Kirchhausen, and A. L. Horwich, "Multivalent binding of nonnative substrate proteins by the chaperonin GroEL", Cell, vol. 100, pp. $561-573,2000$.

(C) Shukla and Paul; Licensee Bentham Open.

This is an open access article licensed under the terms of the Creative Commons Attribution Non-Commercial License (http://creativecommons.org/licenses/by-nc/3.0/) which permits unrestricted, non-commercial use, distribution and reproduction in any medium, provided the work is properly cited. 